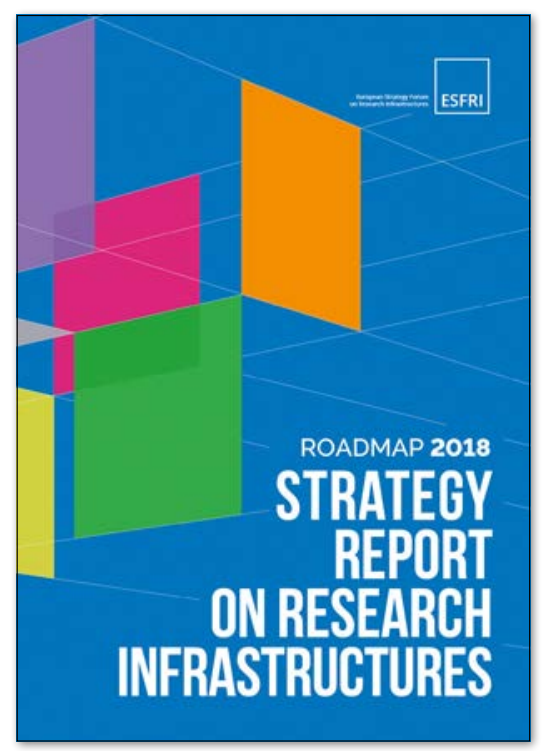

\title{
RESEARCH INFRASTRUCTURES AS A KEY OPTIMIZER OF EUROPEAN RESEARCH
}

n Giorgio Rossi - ESFRI Chair 2016-2018 - DOI: https://doi.org/10.1051/epn/2019101 - Dipartimento di Fisica dell'Università di Milano, via Celoria 16, 20133 Milano, Italy

\section{The European Strategy Forum on Research Infrastructures (ESFRI) has been} created to advise the Competitiveness Council of the European Union on the needs and opportunities to build a strong Research Infrastructure (RI) system, covering all domains of research, providing the most advanced tools that cannot be realized at national level, and that must be accessible to all strongly motivated researchers in order to increase the European science competitiveness.

VFIG. 1: The ESFRI Rls of different level of organization covering specific areas in the Health

\& Food domain.

\section{The ESFRI Forum}

Scientific and ministerial delegations from the $28 \mathrm{Member}$ States and the 12 Associated Countries form the Forum, that has structured its activities with six Strategy Working Groups on the research areas of Energy, Environment, Health \& Food, Physical Sciences \& Engineering, Social \& Cultural Innovation, Data \& Computing \& Digital Research Infrastructures, and one Implementation Group, all made of experts proposed by the Forum. Ad hoc Working Groups of Experts are created by ESFRI, with a focussed short-term mandate, when they are needed.

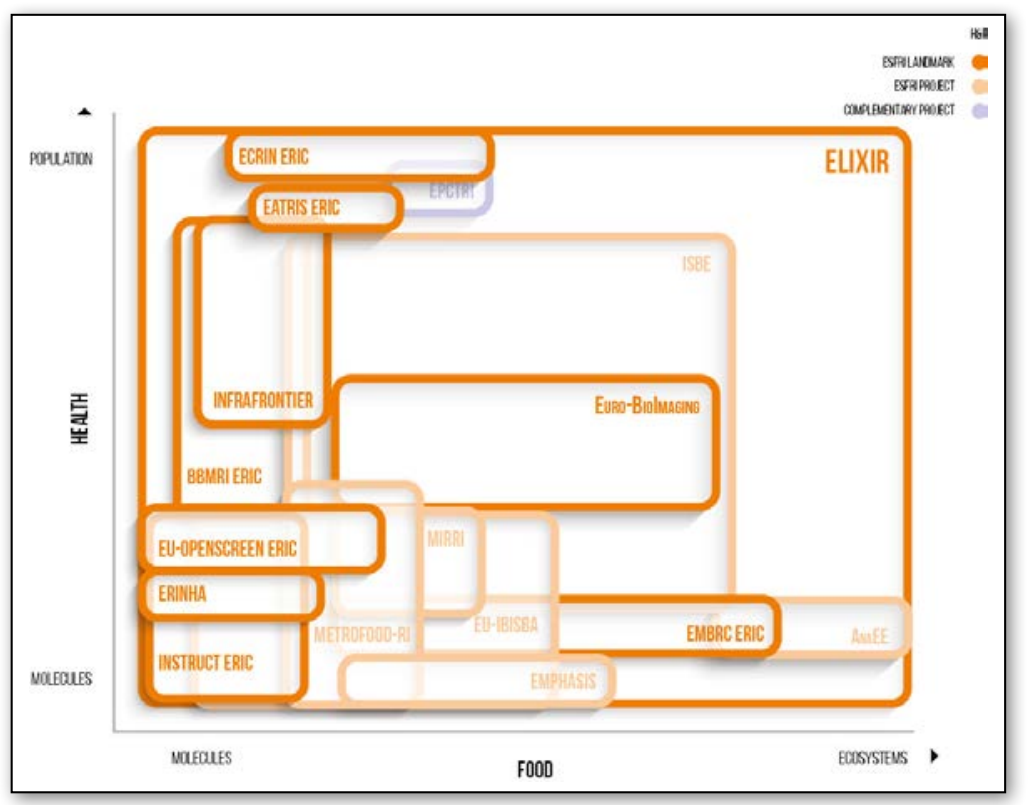

\section{The ESFRI Roadmaps}

ESFRI has produced three roadmaps in the years 20062010 that identified a set of about 50 RIs representing the state-of-the-art in the respective fields, as well as global undertakings in some cases, these were the "orange-cover" roadmaps. Then the Competitiveness Council mandated ESFRI to adapt its action to the goals of the Innovation Europe programme, and to help the implementation of at least $60 \%$ of the roadmap infrastructures by 2016 . This was done through assessments (the European Commission organized the High-Level Assessment Expert Group) and by monitoring the state of play of the RIs, and optimizing a special support programme under $\mathrm{H} 2020$.

The blue-period started with the blue-cover Roadmap 2016 that introduced important new rules and methodology including firstly a political support threshold for eligibility, and secondly clear implementation targets, like the ten-year rule for new Projects to achieve full maturity. The status of Landmark was introduced to identify those successfully implemented RIs that are engaged for a longterm operation at the forefront of their research domain, and to emphasize the need of continuous support for producing the maximum return on investment.

New projects are adopted in the roadmap after a selection process that addresses both the scientific merit of the RI proposal, its uniqueness and pan-European dimension, and its organizational structure (governance, legal status, solidity of multi-national consortium, financial 


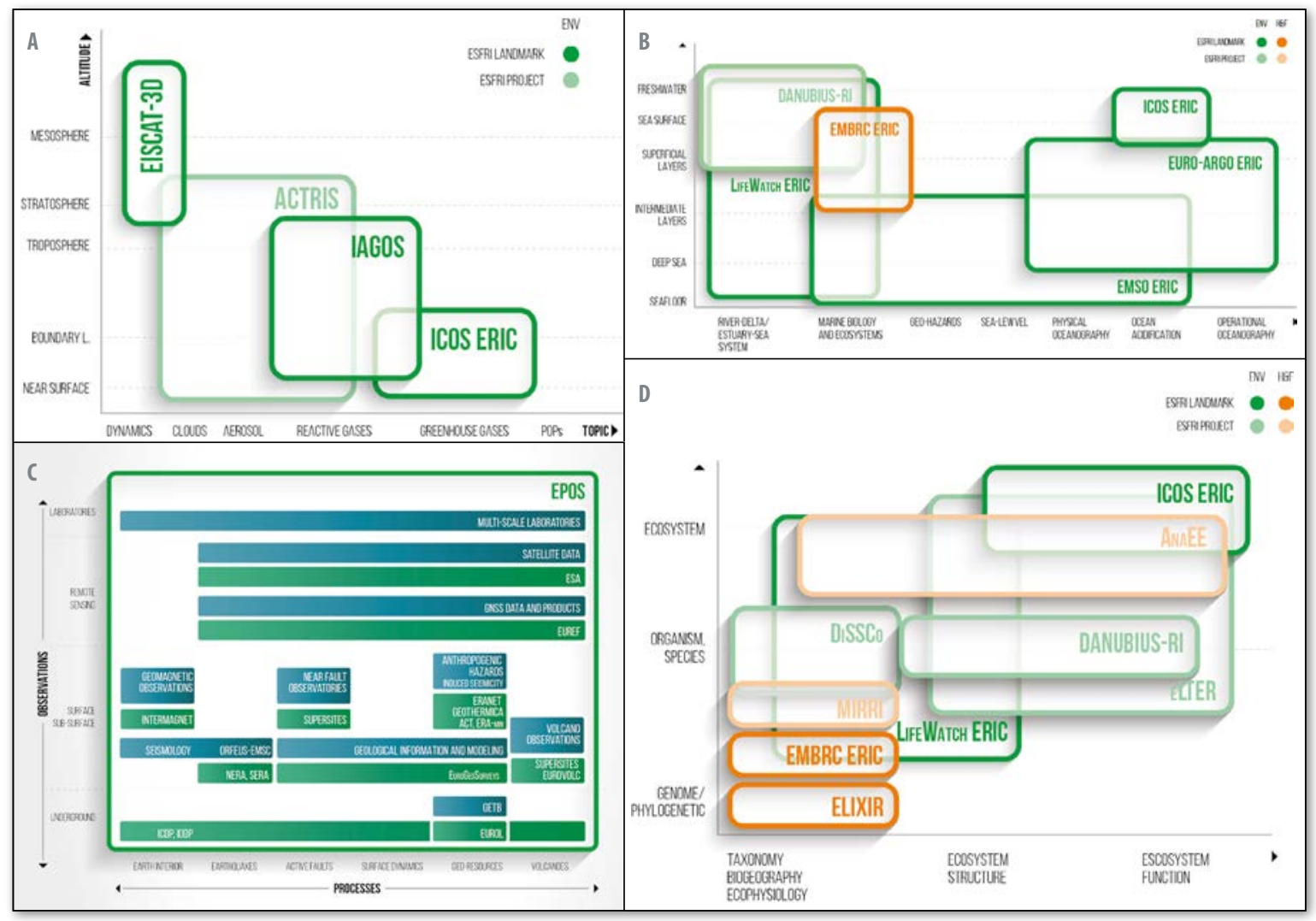

commitments, human resource capital and access rules for the users community). Once enlisted by ESFRI the RI Projects have a maximum of ten years to reach the condition of fully mature implementation-ready infrastructure.

\section{The ESFRI Landmarks}

The Landmarks are RIs that offer openly accessible science services or support direct access to the resources for advanced research by all European and international researchers, with priorities set by peer-review of the scientific proposals. The lifecycle description of RIs, from conceptual outline to full operation and eventually termination, has been adopted by ESFRI to rationalize the objectives and performances expected by the RIs at any given stage of their life.

The Roadmap 2016 listed 21 Projects and 29 Landmarks, including two EIROforum upgrade projects that had already been approved from their Councils: the High Luminosity Large Hadron Collider of CERN (HL-LHC) and the Extreme Brilliant Source of the European Synchrotron radiation facility (EBS-ESRF).

\section{The Landscape Analysis}

The Roadmap 2016 was complemented by an extensive Landscape Analysis (LA) carried out as a comprehensive recognition of the national RIs that operate in Europe with international open access in each of the scientific areas identified by ESFRI, as well as an analysis of the trends of their development, of the outlook elaborated by the scientific societies, and a gap-analysis pointing to the weaknesses of the overall landscape. The LA and the individual descriptive cards of each Project and Landmarks were published in the new www.ESFRI.eu web-site, whilst only the introduction, concept and list of Research Infrastructures were published in hard copy and presented in a dedicated event on $10^{\text {th }}$ March 2016 in Amsterdam.

The new ESFRI Roadmap 2018 was presented in Vienna last September $11^{\text {th }}$, before the International Conference on Research Infrastructures (ICRI-2018), representing the European analysis and contribution to the realization of open access Research Infrastructures for the benefit of European and global science, including all its parts, endorsed by the Forum, in a hard-copy volume.

The new Project list counts 18 initiatives, including 6 new entries in the Energy (ENE), Environment (ENV), Health \& Food (H\&F) and Social \& Cultural Innovation (SCI) domains; the Landmark list counts 37 operational Research Infrastructures showing that most, albeit not all, ESFRI projects complete successfully their incubation towards implementation within the ten years on the Roadmap. The science domains populate the ESFRI list with a number of initiatives that reflect the development of global science.

\section{The strategic domains}

The Health \& Food domain counts ten Landmarks and six Projects, all distributed infrastructures that in fact integrate a large number of national nodes in a strategically driven federation coordinated by a unique, jointly participated, hub that becomes both the science policy maker and the single access point for the users proposals that are then performed
4 FIG. 2: The ESFR RIs covering specific areas in the environmental research domains: A) atmosphere, B) hydrosphere, C) geosphere D) biosphere 


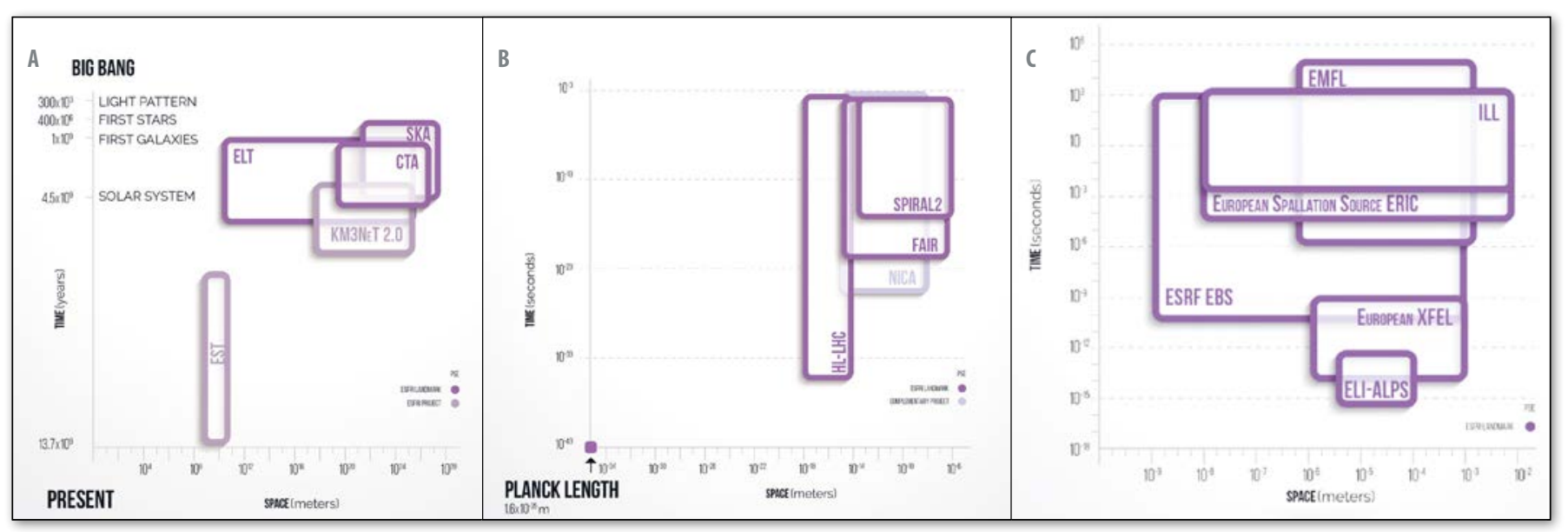

$\triangle$ FIG. 3:

The ESFRI RIs

covering specific areas in the Physical

\& Engineering

domain: A)

Astronomy and Astroparticle Physics,

B) Particle and

Nuclear Physics, C) Analytical Physics. at the most suitable national nodes, with a variety of access modes. The ensemble of the H\&F Landmarks addresses the health challenge, covering from molecule to ecosystem and to population dimensions, as well as the food challenge again from molecular scale to ecosystem and to economy. The ensemble of targeted Landmarks cover specific research needs and addresses, with state-of the-art methods and resources, the big societal challenges. Much of the elaboration by ESFRI on the optimization of the distributed infrastructure model has benefited from this domain and has fed back to it as well as to the whole roadmap more generally. A general bioinformatics infrastructure is providing operational interfaces to all the Landmarks of the domain and is acting as a reference to accelerate the optimal data flow among them, and contributes to set standards for an effective open-science/open-data paradigm (See Figure 1).

The seven Landmarks and four Projects in the Environment domain are also all distributed infrastructures as their research is performed in dedicated observatories as well as on integrated observational data from a very broad array of national and international observatories that are present in the European and world territory with scopes merging science and environment control, including RIs and civil protection national services. The fine structure of the Landmarks in this broad and strategic domain can be described as research on the atmosphere, from near ground to near space, on the hydrosphere from freshwater to marine, on the biosphere covering biodiversity and

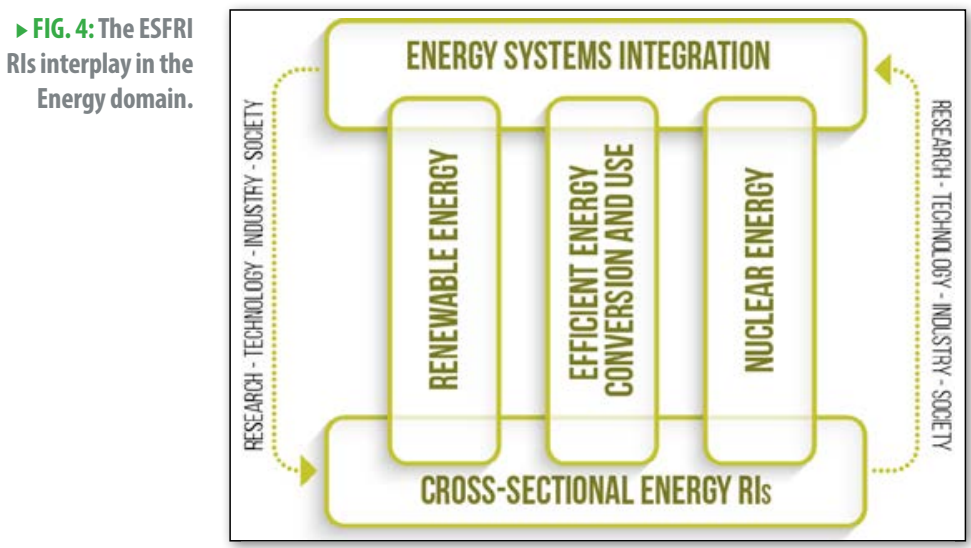

ecosystems, and on the geosphere from the earth surface to the interior of the planet covering geo-hazards and geo-resources. Large data management and advanced data services are developed by the RIs, coping with all the dimensions of the research and the data flow among them in ENV and across disciplines (See Figure 2).

The Social \& Cultural Innovation sector has five Landmarks and two Projects, again all distributed, addressing the integration of big social data from research through longitudinal and horizontal surveys on the ageing of the European population and on the social behaviours of the population and evolution of their institutions; enabling access by all researchers to advanced social data archives; addressing the language commons across all the idioms of European culture and their meanings; addressing the digitalization of the cultural heritage of arts and humanities, the research, elaboration and memory of the holocaust, and the novel concept of heritage science that exploits the most advanced scientific analytical approach to understand the make and materials of the richest cultural heritage on earth, and to develop science-based preservation strategies.

The Physical Sciences \& Engineering domain counts twelve Landmarks and two Projects that are mostly unique and central research facilities, installed in a single site or in a few interconnected sites, addressing fundamental research in astronomy, astrophysics, and on the nature of matter from high energy particle and nuclear physics to the fine analysis of materials. These RIs include major ground based observatories of the universe and large installations to probe the intimate structure of matter, as well as the broadly interdisciplinary research based on advanced analytical probes like X-ray sources (synchrotron radiation, free electron laser, extreme performance laser) and neutron radiation (nuclear reactor and spallation source) as well as installations allowing to reproduce extreme conditions for probing matter in very high magnetic fields. The research needs for observatories of the universe require to build and combine installations from different observation points on the earth, e.g. in both hemispheres, as well as the extreme specialization 
of laboratories built in different sites for sustainability and development of regional scientific communities. The multi-messenger research method has been prompted by the observation of the gravitational waves (GW) and the correlated observations by all electromagnetic-field (EM) observatories at all wavelengths and astroparticle observatories. The real-time response to GW observation events by all other astronomy and astrophysics observatories has major implications on the technology for synchronous operation and on the data transfer and analysis methodologies. Similarly probing complex materials by broad energy range photon, electron, neutron, muon, ion beams and advanced computational simulations converge into a multi-messenger approach to the understanding of aggregated matter (See Figure 3).

Two Landmarks and four Projects in the Energy domain cover the needs of research on materials for energy applications and the methodologies for renewable energy production with large central installations as well as the study of global challenges as the carbon dioxide capture and storage based on a distributed set of experiments (See Figure 4).

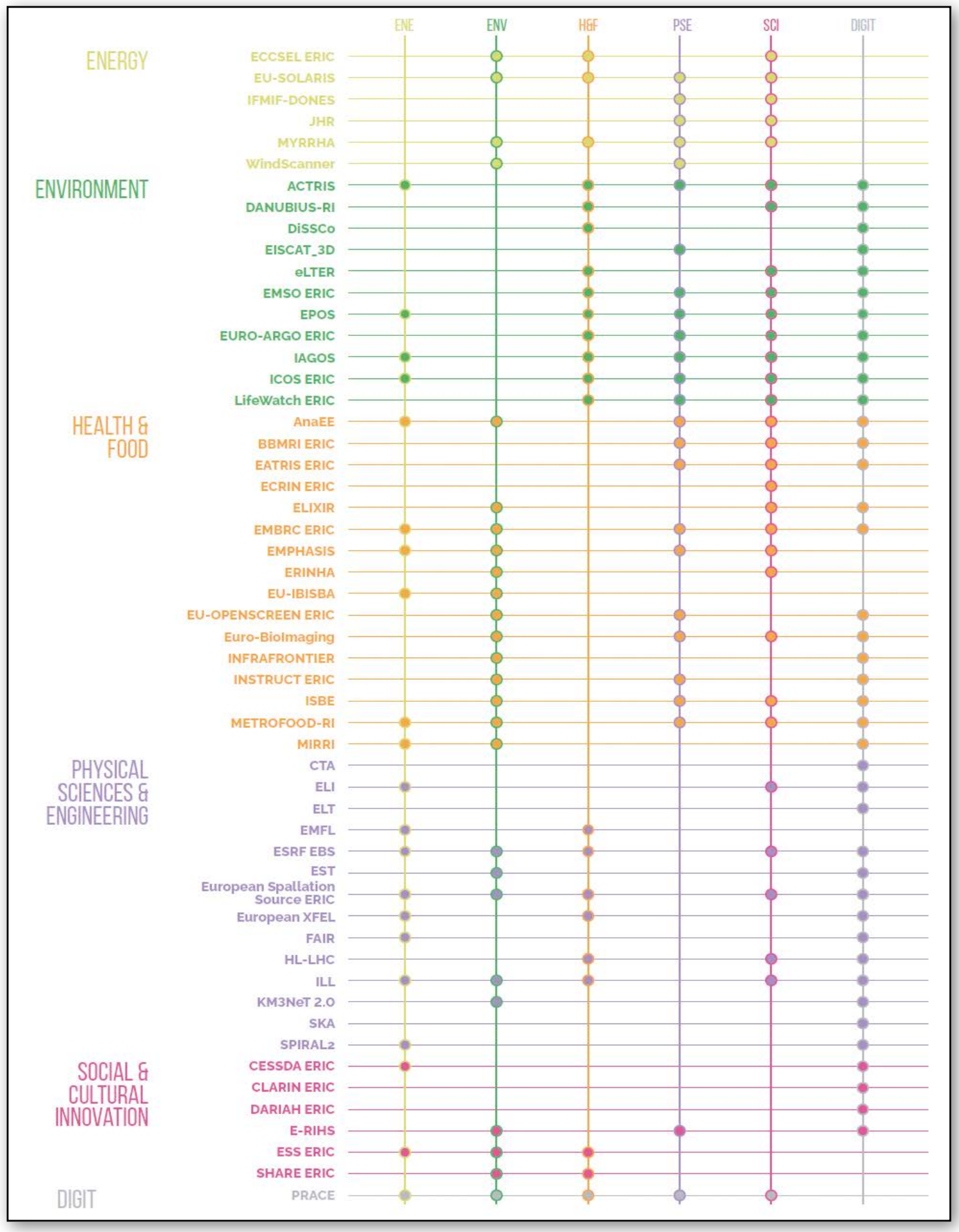

$\checkmark F I G .5$ : The ESFRI RIs Interconnections with different scientific domains 
Finally the Data \& Computing \& Digital Research Infrastructures domain identified one Landmark for the interdisciplinary use of advanced computation organized as one large distributed infrastructure representing the European federated effort in supercomputing with a hierarchical structure of powerful, world standard, computing resources.

\section{The interconnections, beyond the disciplinary paradigm to address global issues}

Knowledge develops across and beyond the disciplinary paradigm that still dominates European culture and high education institutions. The ESFRI Roadmap 2018 gives evidence on the actual impact of the Research Infrastructures on other domains that enlarge their significance well beyond the scientific community that has originally prompted their need. A multi-messenger approach is becoming ubiquitous in science, and a well interconnected RI system is the tool for developing it.

The analysis of the interconnections between the ESFRI RIs and all scientific domains helps in capturing the general significance of a well structured European RI system for pursuing novel scientific knowledge as well as to enable multidisciplinary research efforts. This is in fact one of the challenges for the development of the European Research Area: to become a powerful research system capable to address the urgent needs of new knowledge for coping with the criticalities in the evolution of the planet and of humanity, as well as to effectively pursue solid understanding of life and the universe.

The interconnections represent also the clear need for effective information exchange through high quality data and data services that could also scale up to a general system for accessing scientific knowledge and to a system capable of pursuing the knowledge needs when these are formulated in a non-disciplinary way, as it is the case for the so-called major challenges that humanity faces that are intrinsically complex with tangled implications on energy needs, environment effects and resources, social development and understanding, quality food supply and better health for all people, yielding a more advanced understanding of the concept of global sustainability.

The general issues to which the RIs can, and do, contribute are also reflected in the LA: education and training are within the scope of all ESFRI RIs and it is high time to address the complementarity with the higher education system, e.g. by introducing formal credits; innovation in economic and social activities has a motor in the RIs, but it also depends on improving the knowledge transfer process to the social and economic actors; the socio-economic impact of RIs is to be understood at all relevant levels starting from the optimization of the science production, e.g. of the overall research budget, to the local economy when single site large infrastructures and large hubs contribute to, or even shape, the regional development. Scope of the RIs is the global competitiveness of European research, through shaping international collaborations on major research undertakings as well as playing a prime role in science diplomacy and in the diffusion of scientific knowledge and trustable data.

ESFRI receives additional specific mandates from the Council about RIs, with requests ranging from developing methods for monitoring performance of all Research Infrastructures, to the analysis and strategies of their sustainability, to the overall alignment of scope and methodology with national roadmaps, to contributing towards the making of the European Open Science Cloud as a key instrument for Open Science. ESFRI addresses these mandates, also in good collaboration with the European Commission, and the results of the work by the Forum and by the ad hoc expert groups can lead to publications, the ESFRI Scripta, that diffuse the results of strategy analysis on specific issues.

The results achieved by ESFRI and represented in the ESFRI Roadmap 2018 are broadly acknowledged. This implies that it is now urgent to refine a strategy for the future shaping its broad mission and continuously adapting its procedures to cope with the new mandates and goals. The reflection on the new challenges has started in the Forum and will have a first dedicated plenary workshop in January 2019, under the leadership of the new Chair and Executive Board.

ESFRI has proven to be a truly pan-European effort involving both scientific communities and ministerial authorities of all Member States and Associated Countries and its results demonstrate the high merit and great potential of European collaboration in developing visions and sustainable perspectives for progress, in the spirit of freedom and of the common aim for reaching advanced synthesis and ambitious goals for science and society.

\section{About the Author}

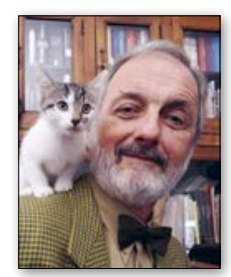

Giorgio Rossi is Professor of Physics at the Università degli Studi of Milano. Italy. He leads the APE (Advanced Photoelectric Effect Experiments) and NFFA (Nano Foundries and Fine Analysis) groups at CNR-IOM and Elettra in Trieste, performing research on the electronic and magnetic properties of surfaces and nanostructures, also operating synchrotron radiation spectroscopy and in situ materials growth facilities open to academic and industrial users. He is author or co-author of over 220 research papers in ISI journals.

He coordinates the NFFA-Europe (Nano Foundries and Fine Analysis) integrated infrastructure since 2008. He has chaired the Physical Sciences \& Engineering Strategy Working group of ESFRI in 2013-2016, and served as ESFRI Chair since $1^{\text {st }}$ July 2016 to $31^{\text {st }}$ December 2018. 